\title{
LA ACCESIBILIDAD DE LA HOYA ALCOYANA DURANTE EL SIGLO XIX
}

\author{
Jesús Rafael de Vera Ferre
}

\begin{abstract}
RESUMEN
La hoya de Alcoy ha tenido siempre serios problemas técnicos y financieros para desarrollar una malla de transportes que funcionara eficientemente debido a su morfología. Este hecho dificultó la entrada de las materias primas y la salida de las manufacturas de su importante industria a lo largo de todo el siglo XIX, incentivando la búsqueda de soluciones que mejoraran la accesibilidad por ferrocarril y carretera.
\end{abstract}

\begin{abstract}
The «Hoya de Alcoy» (Alcoy valley) has always had serious technical and financial problems to develop transport systems that worked efficiently due to its morphology. This fact made difficult the entrance of raw materials and exit of manufactured articles of its important industry during all ninetieth century, incentivating the search of solutions that improved railway and road accessibility.
\end{abstract}

\section{Una red viaria signada por la morfoestructura}

La accesibilidad de las comarcas interiores de L'Alcoià y El Comtat ha encontrado multisecularmente un serio obstáculo en la orografía, lo que ha limitado la mejora de las redes viarias, a la par que encarecía notablemente todo intento de aliviar la situación, puesto que el gran número de obras de fábrica a realizar convertía en poco menos que inviable cualquier tipo de intervención generalizada sobre la malla circulatoria.

Sin duda, la configuración morfoestructural de la zona ha introducido graves dificultades de funcionamiento eficaz y de sistematización organizativa en los flujos circulatorios de pasajeros y mercancías, ya que el sólido cíngulo montañoso que la cerca provoca un aislamiento forzoso muy difícilmente salvable con el instrumental tecnológico utilizado en el país durante centurias, al que siempre se han unido endémicas carencias de los fondos públicos nacionales y locales disponibles para llevar a cabo, con éxito, una renovación de los canales de transporte imprescindibles para que la industria pudiera afrontar competitivamente el futuro. 
En efecto, los caminos presentan un recorrido fuertemente signado por la frecuente alineación SW-NE de las serranías subbéticas. Solamente, el recorrido de Játiva a Alicante, por Alcoy, rompe este esquema lo que propicia el que se convierta en el organizador principal de la red circulatoria al que van a desembocar los demás corredores, siempre afluentes al central. Por el norte, la vía aprovecha para penetrar en la cubeta el desgarramiento de los pliegues de la falla del puerto de Albaida, mientras que al mediodía remonta el de la Carrasqueta de forma penosa.

El resto de los caminos principales se instalan sobre los materiales calizos de las fracturas transversales y el encajamiento de la red hídrica. Tal es el caso de la salida a la Hoya de Castalla, en dirección a Ibi, o el camino hacia Pego encauzado por una angosta morfología hacia el valle de Gallinera. Por su parte, el obstáculo de la sierra Mariola, hacia occidente, es salvado por la red caminera utilizando el valle de Polop y del Barchell en el itinerario hacia Bañeres y el de Agres para el recorrido hacia la población del mismo nombre.

La vialidad de la zona, seriamente comprometida por la realidad morfoestructural, se veía agravada por el clima, con una pluviosidad superior a los $500 \mathrm{~mm}$. anuales, frecuentes heladas y nieves que impedían, a veces, o lastraban, otras, el lento discurrir de los carros, debido a las fuertes pendientes a salvar y al depósito de derrubios y restos vegetales que la escorrentía depositaba sobre el trazado de los caminos, dado el alto poder erosivo de las aguas por su carácter espasmódico y por los fuertes desniveles de las vertientes. Frecuentes cortes de las vías por aterramientos, desprendimientos de la calzada y la existencia de barro impedían el normal desenvolvimiento de los abastecimientos de alimentos y de carbón y la salida fluida de los productos manufacturados textiles y papeleros.

\section{La cubeta alcoyana, un área multisecularmente transitada}

A pesar de las dificultades descritas, la cubeta alcoyana y su alfoz funcional han tenido multisecularmente un cierto nivel de protagonismo en el conjunto de las comunicaciones del Sur del País Valenciano, hecho que hunde sus raíces, sin duda, en la necesidad de acortar el trayecto desde Valencia a Alicante, evitando el rodeo por el valle del Vinalopó o sorteando el dificultoso y tortuoso camino litoral de La Marina, a lo que hay que unir la antigua tradición artesanal de la zona.

El Camí Major de Sexona ya era utilizado en el medioevo, siendo descrito el estado de la ruta en el Cuatrocientos y en el Quinientos por Müenzer ${ }^{1}$ y Villuga ${ }^{2}$ respectivamente. Por su parte, monsieur Des Essart en enero de 1660 hacía la siguiente aseveración:

\footnotetext{
«El camino de Lixona a Coyst es muy malo. Se sube por la misma Carrasqueta y hay que bajar el monte San Antonin que es todo un precipicio y muy elevado».3
}

\footnotetext{
${ }^{1}$ MUENZER, J., «Relación del viaje por España», apud GARCÍA MERCADAL, J., Viajes de extranjeros por España y Portugal. Madrid, Aguilar, 1952, t. I, pp. 327-418. El viaje lo realiza entre 1494 y 1495.

${ }^{2}$ VILLUGA, P. J., Repertorio de todos los caminos de España hasta agora nunca visto el qual allaran cualquier viaje que quieran andar muy provechoso para todos los caminantes. Medina del Campo, 1546. Utilizada edición facsímil, Madrid, Reimpresiones Bibliográficas, 1951.

${ }^{3}$ DES ESSART., «Journal du voyage du Sieur des Essart», apud BERTAUT, F., Journal du voyage d'Espagne. Paris, Louis Billaine,1679, p. 241.
} 
En el siglo XVIII, Cavanilles nos indica que existían fundamentalmente seis caminos en la zona alcoyana. El más importante, el que une Valencia con Játiva, Alcoy, Jijona y Alicante, mientras que las comunicaciones con el valle del Vinalopó, y, desde allí, con Murcia y Andalucía, se establecían por el valle de Agres, en la ruta a Bañeres, y, por la Hoya de Castalla, con dirección a Ibi, Biar y Villena. Por su parte, los enlaces orientales discurrían, desde Cocentaina, por un camino de herradura que se bifurcaba en un doble ramal, uno, orientado hacia el valle del Seta, por Benifallim, Penáguila, Agres, Gorga y Tollos; el otro, fluía hacia el valle de Gallinera por Benimarfull, Planes, Benitaya y Carroja para terminar en $\mathrm{Pego}^{4}$.

\section{La estrategia de la clase dirigente ochocentista en el tema del transporte}

Dada la situación de utilización constante de una red de transportes totalmente inadaptada a las necesidades básicas de las actividades industriales desarrolladas en la comarca, no es extraño que una de las mayores preocupaciones de la clase burguesa alcoyana fuera el intentar resolver el déficit de infraestructuras, utilizando para ello las influencias políticas necesarias a nivel estatal $\mathrm{y}$, sobre todo, provincial. Sin embargo, la solución a este problema capital siempre quedaba aplazada o mal resuelta, debido a lo costoso de las obras, dada la dificultad que entrañaba el adaptar el aparato circulatorio a la movida morfología, diseñando los trazados en abruptas vertientes, a la par que se hacía imprescindible el salvar importantes desniveles y los lechos de los ríos y barrancos, siempre propensos al desbordamiento, lo que suponía la realización de obras de ingeniería de gran magnitud. No menos necesario era el mantener en conservación lo realizado, frecuentemente destrozado por las lluvias o cuarteado por el hielo y la nieve, al tiempo que siempre quedaba el problema de las acusadas pendientes, los desprendimientos y las curvas cerradas.

La burguesía ochocentista, a pesar de su afán innovador y de su espíritu de empresa, va a mostrarse impotente para dar una solución definitiva al problema de la accesibilidad a la hoya de Alcoy durante la centuria, ya que el haz de necesidades básicas era variado al unirse importantes déficits de agua y de energía y tener que hacer frente a la feroz competencia del textil catalán que, gracias a la mejora de los sistemas generales de transporte, veía ampliada su influencia y sus mercados en mayores porciones del territorio nacional. En ese sentido, Barcelona, puerta española de Europa, contaba con una gran ventaja, ya que paulatinamente acumulaba infraestructuras de transporte con lo que supone de inducción hacia una economía de escala, mientras que Alcoy quedaba, cada vez más, al margen de lo que con el discurrir del tiempo habrían de constituirse en los grandes canales axiales de los ferrocarriles y de las carreteras estatales.

En la estrategia global del transporte, a los empresarios alcoyanos les interesaba actuar en varios frentes lo que sin duda producía una no deseada dispersión del esfuerzo, en un momento, como es el de los albores del Alicante isabelino, en que casi todo estaba por realizar en el campo de las comunicaciones a nivel endógeno y exógeno, a la par que la

\footnotetext{
${ }^{4}$ CAVANILLES, A. J., Observaciones sobre la Historia Natural, Geografía, Agricultura, Población y Frutos del Reyno de Valencia. Madrid, Imprenta Real, 1792. Utilizada edición facsímil, Valencia, Gráficas Soler, 1972 , v. II, pp. 13 y ss. Esta misma información se reproduce casi íntegra a mediados del Ochocientos por MADOZ, P. Diccionario Geográfico-Estadístico-Histórico de España y sus posesiones en ultramar. Madrid, Tip. Pascual Madoz, 1845-1850, 16 vol. Se ha utilizado la ed. facsímil, en 2 vol., referente al Reino de Valencia. Valencia, Institución Alfonso el Magnánimo, 1982, t. I, pp. 6 y 42.
} 
burguesía de la ciudad capital luchaba denodadamente por consolidar su rol de organizadora del territorio atrayendo hacia la urbe lucentina los principales ejes ferroviarios y de carreteras, con indudable beneficio para sus instalaciones portuarias al tiempo que se consolidaba su influencia política.

Para la industria alcoyana era vital el poseer unos enlaces con el litoral en buen estado, ya que por los puertos de Gandía y Alicante desembarcaba gran parte del carbón alóctono utilizado en su actividad, el cual era transportado, mediante carros, con no poca dificultad, hacia las instalaciones fabriles de la hoya. También se consideraba interesante el tener buena conexión con la Meseta y la capital del Reino, máxime cuando buena parte del textil fabricado iba destinado al ejército, y con Murcia y Andalucía donde, posiblemente, podía jugar con una cierta ventaja inicial respecto a Cataluña en la ampliación del mercado, dada la mayor proximidad a la zona y las deficientes comunicaciones existentes con Barcelona en el mediodía del país, lo que se traducía en unos mayores costos de transporte.

El enlace con la ciudad de Valencia era también conveniente y no mayormente por la utilización de su puerto, aunque éste también fuera un atractivo, sino especialmente porque esta urbe se convertía en el enlace con Aragón y, a través de él, con el Norte de España, y con Cataluña y, por ende, con Europa.

Por último, era también imprescindible un cierto alivio en la situación de los caminos que conducían a La Marina, ya que dicha ruta aseguraba la llegada de subsistencias al mercado alcoyano, especialmente cereal, vino, aceite, almendras y frutas, ya que la población de la capital del Serpis, juntamente con la de Cocentaina superaba, desde 1860 los treinta mil habitantes ${ }^{5}$, al tiempo que las vías interiores de la comarca tenían que asegurar, aunque fuera mínimamente, el discurrir de los productos en proceso de fabricación, ya que no se debe olvidar que hasta mediados de la década de los sesenta del pasado siglo el hilado y cardado de la lana se hacía, mediante sistema domiciliario, en los pueblos del alfoz, situación que va a saltar por los aires a partir de la implantación de las fábricas en el núcleo capital.

A la generalizada aspiración de mejora de las carreteras y las comunicaciones por parte de la población alcoyana y, especialmente, de su clase dirigente, se unía el creciente interés de la burguesía mercantil alicantina de atraer hacia el puerto de la capital la entrada de materias primas y del carbón y de salida de las manufacturas de la hoya del Serpis. Por ello, no es de extrañar que la prensa lucentina hiciera constante alusión a la necesidad de construir un ferrocarril que uniera ambas ciudades, tal como queda de manifiesto, por ejemplo, en El Graduador ${ }^{6}$ en 1877, al tiempo que la opinión pública de la Montaña urgía la realización definitiva de la carretera un año más tarde ${ }^{7}$, una ruta vital que, aunque, a principios de la centuria, era ya de $\left\langle\right.$ rueda ${ }^{8}$ se encontraba en un pésimo estado ${ }^{9}$ y en situación muy lejana a lo que era una vía importante en el Ochocientos.

La clase dirigente alcoyana, aun sin abandonar sus aspiraciones a mejorar sus caminos y carreteras, secularmente abandonados, puso sus mayores esperanzas en la consecu-

\footnotetext{
${ }^{5}$ PÉREZPUCHAL, P., «La población del País Valenciano hasta la época estadística», Cuadernos de Geografía, 10, 1972, pp. 1-30.
}

${ }^{6}$ El Graduador, de 20 de mayo de 1877 urgía la realización del ferrocarril Alcoy-Alicante. Ya antes, en 1868, tal como informaba El Comercio de Alicante, de 5 de abril de 1868, se buscaba una fórmula para llevar a cabo la línea férrea de Villena-Cocentaina-Alcoy.

${ }^{7}$ Tal como refleja El Serpis del 9 de mayo de 1878.

${ }^{8}$ Según la opinión de LÓPEZ, S., La nueva guía de caminos. Madrid, 1812, p. 161.

${ }^{9}$ MIÑANO, S., Diccionario Geográfico-Estadístico de España y Portugal. Madrid, Pierart-Peralta, 1826-1829, t. I, pp. 107 y ss. 
ción de varios enlaces ferroviarios, ya que el tren era el modo de transporte principal de la centuria saldándose la situación con un estrepitoso fracaso, ya que de las distintas opciones manejadas, cifradas en la conexión con Alicante; el corredor del Vinalopó, a través de Villena, enlazando desde la misma con el altiplano de Yecla; Játiva y Gandía, solamente fructificó en el Ochocientos este último, diseñado como de vía estrecha, y con la finalidad principal de importar carbón del Reino Unido, que fue abierto al público el veinticinco de enero de 1893.

Por otra parte, a nivel político, existía mucho interés en solucionar los enlaces de Alcoy con la capital provincial como una forma de estructurar la nueva demarcación administrativa, mediante el drenaje de nuevas zonas que amplificaran el rol de la urbe alicantina con un espacio diseñado artificialmente desde Madrid y que presentaba elementos divergentes y centrífugos importantes, con fuerte atracción de ciertas comarcas por parte de algunas provincias colindantes. Por ello, era vital para la Diputación el que los dos polos más activos económicamente del Sur del País Valenciano quedaran unidos por los intereses comunes que dimanan del transporte, el comercio y la circulación de personas. Esta es la consigna que se enarbola constantemente a lo largo de la centuria y que nunca fue totalmente resuelta como queda patente todavía hoy, a fines del siglo XX, cuando aún se sigue planteando la necesidad de dotar de un túnel a la Carrasqueta y empiezan a ponerse las bases para mejorar el trazado, mediante la construcción de variantes, en la coalescencia formada por San Juan y Mutxamel en la Huerta de Alicante. En este sentido, se puede afirmar, sin miedo a equivocarse, que hasta que no exista una comunicación fluida de la ciudad capital con la canal de Jijona y la cubeta alcoyana no se habrá acabado de fraguar definitivamente la integración de las tierras meridionales valencianas.

\section{Actuaciones en materia de carreteras en el período isabelino}

\subsection{El importante eje Játiva-Alcoy-Alicante: problemas y realizaciones}

Aunque el estudio de posibles soluciones a la escasa accesibilidad del Alcoià-Comtat son anteriores, las primeras decisiones datan del período isabelino, época pionera, donde, sin embargo, priman más las buenas intenciones que la terminación de las obras, que se dilataban de forma extraordinaria, hecho que hunde sus raíces tanto en la penuria endémica de los presupuestos como en la propia dificultad del terreno. El capitán Cook Widdrington indicaba en 1834 que aunque se había iniciado la brega en el tramo Játiva-Alcoy, los trabajos estaban totalmente paralizados por falta de fondos ${ }^{10}$, mientras que Nicasio Camilo Jover nos dice que dos años más tarde se consigna un presupuesto por parte del Gobierno de la Nación para el tramo indicado ${ }^{11}$.

En el año 1842, con el mismo fin de recaudar fondos para todo el itinerario hasta Alicante, se dispuso que el impuesto de cuatro maravedíes por carta que se pagaba en la provincia lucentina para contribuir a la construcción de la carretera de las Cabrillas se

\footnotetext{
${ }^{10}$ WIDDRINGTON, S. E. C., Sketches in Spain during the years 1829, 30, 31 and 32; contaigning notices of some districts very little known; of the mannors of the people, government, recent changes, commerce, fine arts, and natural history. Paris, Galignani-Baudry, 1834, t. I., p. 28.

${ }^{11}$ JOVER, N. C. Reseña Histórica de la ciudad de Alicante. Alicante, Viuda de Carratalá, 1863, p. 178. El citado presupuesto fue aprobado por Real Orden de 4 de octubre de 1836 con cargo a los presupuestos del año siguiente.
} 


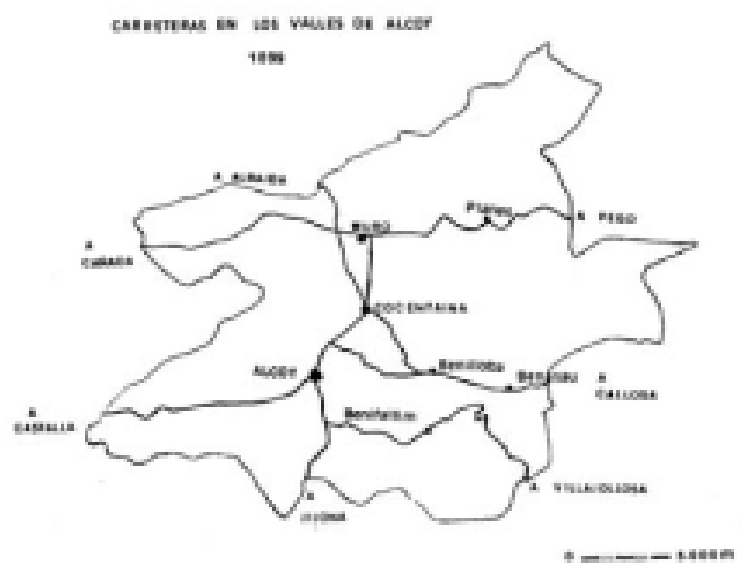

Mapa 1

destinase a las obras del nuevo eje, al tiempo que la Diputación instaba a la de Valencia para ponerse de acuerdo sobre la intensificación de los trabajos para paliar el paro en aumento alentado por la continuada sequía ${ }^{12}$.

A pesar de las buenas intenciones, el ritmo de las obras era extremadamente lento, de tal forma que a finales de la década de los cincuenta estaba terminándose el tramo entre Cocentaina y Alcoy, cuya dificultad queda de manifiesto si se tiene en cuenta que se realizaron, entre otras fábricas, ocho puentes, agravándose de esta forma la penosa situación del transporte, máxime si se tiene en cuenta que, según la Gaceta de Madrid, las diligencias de Valencia a Alicante, por Játiva y la capital del Serpis, movían 12.000 pasajeros anuales, realizando 1.224 viajes $^{13}$.

La década de los sesenta va a dar un nuevo impulso a la mejora de la accesibilidad. En 1861, se construyen los puentes de la Pechina y de San Roque en el interior de la ciudad alcoyana ${ }^{14}$ con el fin de salvar los barrancos que la constriñen y continúan las obras de la carretera hacia el mar en el sector de la importante falla del barranco de la

${ }^{12}$ Revista de Obras Públicas. Suplemento sobre legislación. Primera serie, t. I, 1855, pp. 91 a 94 y 216 a 222. Es la Ley de 24 de febrero de 1842.

${ }^{13}$ Gaceta de Madrid, del 7 de agosto de 1846.

${ }^{14}$ Con anterioridad, de 1828 a 1837, se había construido en Alcoy el puente de María Cristina. 
Batalla, iniciándose el año antes las obras de la que habrá de unir a la capital comarcal con Villena. Todo ello se producía en un período en el que la industria textil se encuentra en pleno proceso de expansión y renovación, debido al nuevo utillaje de los husos mecánicos movidos por fuerza hidráulica, al tiempo que la industria papelera ${ }^{15} \mathrm{y}$ metalúrgica protagonizaban cambios importantes y notables oleadas inmigratorias, procedentes muchas de ellas del propio alfoz, nutrían fábricas y talleres como mano de obra barata.

Si en el período de 1863-1866 se asiste a una importante agilización del ritmo de la obra de la carretera Valencia-Alicante por Alcoy ${ }^{16}$, a partir de 1868 decae la actividad ${ }^{17}$ abortando así las justas aspiraciones del empresariado local.

\subsection{La larga empresa de la construcción de la carretera del barranco de la Batalla a Villajoyosa}

Mención especial merece el caso de la construcción de la carretera que habría de unir el barranco de la Batalla con Villajoyosa ya que es paradigmática de lo que fue la política de carreteras gestionadas por la Diputación en el período isabelino en el que los proyectos eran utilizados con un marcado carácter electoralista y las obras se dilataban durante largos períodos.

En 1863 se inicia la construcción del primer tramo ${ }^{18}$ prolongándose hasta 1875. Mientras éstas se llevan a cabo, se proyecta el segundo trozo en 1867, entre Penáguila y Seguró ${ }^{19}$, durando las obras hasta $1885^{20}$, mientras que entre 1870 y 1875 se brega

en los sectores cuarto y quinto ${ }^{21}$, al tiempo que es necesario, debido a las lluvias, el afrontar la reconstrucción del trazado anteriormente realizado ${ }^{22}$.

Una idea de la laboriosa realización de la carretera la da el hecho de que ya en nuestra centuria, en 1923, faltan todavía once kilómetros para la total culminación de la vía ${ }^{23}$.

${ }^{15}$ CERDÁ, E. Monografía sobre la industria papelera. Alcoy, Aitana, 1967, p. 114.

${ }^{16}$ Por Real Orden de 24 de mayo de 1864 se aprueba el proyecto del tramo entre el Barranco de la Batalla y la entrada a Jijona. Por otro lado, en el período 1863-1866 se construyen algo más de $25 \mathrm{Kms}$. de carretera entre las provincias de Valencia y Alicante. En la última fecha indicada, hay finalizados casi $61 \mathrm{Kms}$. de los que 38 corresponden a la demarcación valentina y el resto a la lucentina, teniendo proyecto aprobado otros $31 \mathrm{Kms}$. En 1867, se trabaja en $25 \mathrm{Kms}$. repartidos entre las dos provincias, según la Memoria sobre las Obras Públicas en 1867, 1868 y 1869 comprendiendo lo relativo a carreteras presentada al Excmo. Sr. Ministro de Fomento por la Dirección General de Obras Públicas. Madrid, Imprenta y Estereotipia de M. de Rivadeneyra, 1873.

La década de los sesenta supone también la puesta en marcha del Plan de Caminos de la Diputación Provincial (A.D.P.A. Acta del 8 de mayo de 1860) que prevee, entre otros, la construcción del de Cocentaina a Callosa d'En Sarrià, a través de Peñas Águilas y Guadalest, y el de Villena a Alcoy por la Cañada, Campo de Mirra y Bañeres, obra esta última que se inicia enseguida. En cuanto a la primera, al final del período isabelino, en 1868, tenía concluido 38 Kms., restando por finalizar 10. También se trabaja en la de Cocentaina a Denia por Pego, con 7'5 Kms. construidos en la fecha indicada, casi 11 en obras y 34 en estudio. También estaba parcialmente realizada la de Alcoy a Bañeres (ADP. Sección de Vías y Obras, leg. 41, 263 y 289).

${ }^{17}$ Entre 1868 y 1869 solamente se construyen 500 metros en nuestra provincia y 10'5 en la de Valencia. En 1869, se suspenden las obras del tramo Játiva-Bellús, iniciadas dos años antes, ya que el contratista rescinde el contrato por falta de pago. En julio se reinicia la brega. Vid. Memoria de las Obras Públicas en 1870, 1871 y 1872 comprendiendo lo relativo a carreteras presentada al Excmo. Sr. Don Víctor Cardenal, Director General de Obras Públicas. Madrid, Establecimientos Tipográficos de Manuel Mimera, 1875.

${ }^{18}$ A.D.P.A. Sec. V. y O. Leg. 290.

${ }^{19}$ A.D.P.A. Sec. V. y O. Leg. 37.

${ }^{20}$ A.D.P.A. Sec. V. y O. Leg. 326.

${ }^{21}$ A.D.P.A. Sec. V. y O. Leg. 381.

${ }^{22}$ A.D.P.A. Sec. V. y O. Leg. 54.

${ }^{23}$ TORMO, E., Levante. Madrid, Guías Calpe, 1923, p. 251. 


\section{La lentitud de las obras viarias en el sexenio revolucionario}

El movido panorama que configura el sexenio revolucionaria va a afectar negativamente al ritmo de las obras públicas fundamentales para la mejora de la accesibilidad de la cubeta alcoyana. Así, la carretera de Alicante a Játiva permanece hasta 1872 sin actividad constructora, comenzando la brega en el mes de septiembre en el tramo comprendido entre el barranco de la Batalla y Jijona, al tiempo que en la provincia valentina se termina el trozo de Játiva a Bellús que se había iniciado en 1867.

Por su parte, la vía que habría de unir Cocentaina con Denia, a través de Pego, desarrolla una débil actividad, estando paralizada la de Alcoy a Callosa d'En Sarrià, por Penáguila, faltando por estudiar en la misma un tramo de 29'5 kilómetros correspondiente a la zona de Benasau, Confrides, Beniardá y Guadalest.

En la misma situación de paro se encontraba la de Alcoy al confín de la provincia de Murcia, a través de Ibi y Villena, faltando por acabar el tramo de diez kilómetros comprendido entre la capital del Alto Vinalopó y el límite de la demarcación lucentina.

La situación de las carreteras dependientes de la Diputación no era mucho mejor ${ }^{24}$, continuando la lenta construcción de la carretera del Barranco de la Batalla a Villajoyosa ${ }^{25}$ mientras que se afronta la construcción del camino vecinal que uniría Muro de Alcoy con Gayanes ${ }^{26}$ y se pone un mayor énfasis en la conservación del viario existente, reparándose, entre otras, la vía vecinal de Alcoy a Bañeres ${ }^{27}$.

Sin embargo, dadas las circunstancias políticas, el período fue francamente parco en la elaboración de proyectos, destacando el estudio de la carretera provincial de Cocentaina a Benilloba $^{28}$.

\section{La mejora de la accesibilidad durante la Restauración \\ 6.1. Hacia una cierta consolidación de la red}

La Restauración supone la consolidación de la red viaria, con la finalización de líneas axiales importantes, como, por ejemplo, la de Játiva a Alicante o la de Cocentaina a Denia, asegurando la interconexión con los mercados exógenos y la llegada de materias primas para el abastecimiento industrial y de productos agrarios al mercado alcoyano.

$\mathrm{Al}$ darse por finalizada la carretera que une directamente, por Jijona, a la ciudad capital con Alcoy se conseguía una de las mayores aspiraciones de las clases dirigentes de ambas urbes, ya que la penosa situación anterior había lastrado enormemente la circulación general de mercancías, servicios y personas entre las dos plazas, con lo que se frenaban los beneficios para las clases burguesas industrial y mercantil. Este hecho ya había sido denunciado por el periódico El Serpis en 1878, al indicar que la falta de comunicación directa entre el postpaís montañoso y el puerto alicantino suponía un fuerte perjuicio para este último ya que las materias primas necesarias para el complejo fabril de la hoya tenía que provenir desde la provincia valentina ${ }^{29}$.

\footnotetext{
${ }^{24}$ Existe para el período, en el A.D.P.A. un Libro de Registro de documentos de entrada y salida de carreteras provinciales, caminos vecinales y canales de riego de la provincia de Alicante.

${ }^{25}$ A.D.P.A., Sec. V. y O. Leg. 443.

${ }^{26}$ A.D.P.A Memoria de la Diputación Provincial. Manuscrita, 3 de noviembre de 1871.

${ }^{27}$ A.D.P.A. Sec. V. y O. Leg. 38 y 87.

${ }^{28}$ A.D.P.A. Sec. V. y O. Leg. 24.

${ }^{29}$ El Serpis de 9 de mayo de 1878.
} 
Evidentemente, la mala accesibilidad de una arteria axial tan importante perjudicaba también a la burguesía alcoyana, ya que la misma ha jugado un papel secundario, si se quiere, pero real, en la génesis y evolución de ciertos atrasos técnicos que algunos sectores de la industria de la zona padecieron durante toda la centuria. No solamente existía un déficit a la hora de surtirse de fuentes de energía o dificultades en el proceso de acumulación de capital que permitiera una renovación tecnológica, sino también una importante distorsión de los circuitos de transporte y distribución lo que provoca una falta notable de agilidad en los canales comercializadores, lo que se traduce en una elevación creciente de los costos, tanto para la atracción de materias primas como para la colocación en los mercados de los productos manufacturados.

Simultáneamente, los continuos retrasos en la recepción de las mercancías y materias primas, por lo deficitario de la red, que, además, con frecuencia, queda colapsada por las nieves o el tránsito se hace peligroso por la existencia de hielo, agregaban importantes deseconomías en el proceso productivo, limitando extraordinariamente la rentabilidad y la expansión del mismo.

Por ello, la etapa de la Restauración supone un balón de oxígeno a la vialidad de la zona, no solamente ya por la terminación de la carretera a Alicante, sino también por la entrada en servicio de la de Cocentaina a Denia, por Pego, que agiliza el tráfico hacia el Marquesado, y por las mejoras de los enlaces con el Alto Vinalopó y, por ende, con la meseta y el altiplano de Yecla y Jumilla. A ello se une la inauguración, en la década de los noventa, del ferrocarril de Alcoy a Gandía que posibilita un mayor contacto comercial con el puerto de la Safor, lo que redundará en un más rápido aprovisionamiento de carbón, infundiendo savia nueva al entramado industrial de la hoya.

Las mejoras, pues, son evidentes y ello, a pesar de que existían en la prensa de la época serias dudas acerca de que alguna de las carreteras inauguradas, cual es el caso de la de la Carrasqueta, pudiera llevar a cabo, de forma eficiente, su misión, dada la alta accidentabilidad del relieve por el que se había trazado. Así, El Serpis decía, en 1881, lo siguiente:

\begin{abstract}
«... un camino en mala hora ideado por un ingeniero poco celoso de la seguridad de los viajeros y del buen servicio de comunicaciones, por lo cual debería estudiarse la manera de variar dicho trazado, si es que se desea que la citada carretera sea una vía que reuna las condiciones apetecidas y propias de su importante tránsito ${ }^{30}$.
\end{abstract}

Por otro lado, las mejoras viarias coinciden en el tiempo con el auge de la industria alcoyana que se hace especialmente patente desde finales de los setenta hasta la década de los noventa. Así, en 1880, se instala en Alcoy la maquinaria Robert de fabricación de papel continuo en el taller Serra, mientras que, en los últimos años del penúltimo quinquenio del Ochocientos, el textil sufre ajustes importantes en su proceso productivo, al aumentarse la mecanización, con el consiguiente ascenso de la productividad y de los beneficios por mayor volumen de producción y reducción de la mano de obra.

Por otro lado, la carretera que une la hoya con Denia supone, entre otros muchos beneficios, la agilización de la llegada allí del vino elaborado en el postpaís montañoso y en el altiplano de Jumilla y Yecla ya que los caldos existentes en el Marquesado eran escasos y de mala calidad.

${ }^{30}$ El Serpis de 5 de enero de 1881. 


\subsection{Positivo balance del período}

Las obras realizadas durante la Restauración consiguen dar una cierta estructuración al deficiente mapa de carreteras del Alcoià-Comtat. A fines de 1833 se puede considerar definitivamente terminada la vía de Játiva a Alicante ${ }^{31}$, aunque las lluvias producían fuertes daños en su estructura que no siempre eran reparados con prontitud. Aunque en 1877 y 1882 se llevan a cabo obras de mejora ${ }^{32}$, la solución a los destrozos no se afrontó en firme hasta $1888^{33}$.

Por su parte, la carretera de Cocentaina a Denia se finalizaba a fines de siglo, aunque el trazado hubo de ser reparado en $1888^{34}$ porque las tormentas lo habían dañado mucho, destruyendo, incluso, completamente, algunas obras de fábrica.

Mientras tanto, continuaban las obras de la carretera del barranco de la Batalla a Villajoyosa, no terminándose totalmente en el período. Las obras de esta vía fueron lentas desde 1875 a $1877^{35}$, intensificándose en los años siguientes ${ }^{36}$, subastándose el tercer tramo en $1881^{37}$, a la par que los gastos de conservación de lo construido fueron notables ${ }^{38}$, quedando por construir en el alborear del nuevo siglo once kilómetros.

La carretera de Alcoy a Callosa d'En Sarrià tenía finalizados en 1882 algo más de 17 kilómetros ${ }^{39}$, reparándose en ese mismo año el puente sobre el río Penáguila ${ }^{40}$.

Por último, en 1884, se aprobaba el proyecto del tramo de siete kilómetros de la carretera de Fuente la Higuera a Yecla, por Caudete, que impedía la continuidad de la vía ${ }^{41}$ y que, sin duda, habría de redundar, aunque fuera indirectamente, en la mejora de las comunicaciones alcoyanas.

Como conclusión, hay que indicar que al iniciarse el siglo XX, la comarca tiene en pleno funcionamiento la carretera de Játiva a Alicante, la de Alcoy a Villena y la de Cocentaina a Denia, estando en construcción la de la capital del Serpis a Callosa en el tramo hasta Benilloba, contando con proyecto aprobado la sección que desde esta última localidad conduce al collado de Camarell y en estudio el trozo restante hasta la Marina Baja.

${ }^{31}$ OBRAS PÚBLICAS. Memoria sobre el estado de las carreteras en el año 1883 presentada al Excmo. Sr. Ministro de Fomento por el Excmo. Sr. Don Mariano Catalina y Cobo, Director General de Obras Públicas. Madrid, Tipografía de los Huérfanos, 1886.

${ }^{32}$ A.D.P.A. Sec. V. y O. Leg. 259.

${ }^{33}$ OBRAS PÚBLICAS. Memoria sobre el estado de las carreteras en el año 1888 presentada al Excmo. Sr. Ministro de Fomento por el Ilmo. Sr. Don Manuel Pardo y Sánchez Salvador, director general interino de Obras Públicas. Madrid, Tipografía de Ricardo Álvarez, 1889. El presupuesto de reparación se aprobó el 9 de marzo de 1888.

${ }^{34}$ Ibíd.

${ }^{35}$ Las obras de este período están consignadas en el A.D.P.A., sec. V. y O. en el legajo 163 y los proyectos en el leg. 70.

${ }^{36}$ A.D.P.A. Sec. V. y O. Leg. 16, 22, 179, 277 y 298; proyectos de algunos tramos en el leg. 260.

${ }^{37}$ A.D.P.A. Memoria de la Diputación, manuscrita, de 31 de marzo de 1881.

${ }^{38}$ A.D.P.A. Sec. V. y O. Múltiples expedientes de conservación en los legs. 142, 271, 212, 266, 328 y 337.

${ }^{39}$ OBRAS PÚBLICAS. Memoria sobre las Obras Públicas en el año de 1882, comprendiendo lo relativo a carreteras, presentada al Excmo. Sr. Ministro de Fomento por el Excmo. Sr. Don Gabriel Enríquez, director general de Obras Públicas. Madrid, Tipografía Hispano-Americana, 1884.

${ }^{40}$ A.D.P.A. Sec. V. y O. Leg. 99.

${ }^{41}$ OBRAS PÚBLICAS. Memoria sobre el estado de las carreteras en el año 1885, presentada al Excmo. Sr. Ministro de Fomento por el Excmo. Sr. Don José Gallego Díaz, Director General de Obras Públicas. Madrid, Tipo-Litografía de L. Peant e hijos, 1887. 